Summer 2009-07-01

\title{
An Investigation of the use of a High Resolution ADC as a Digital Biopotential Amplifier
}

\author{
Damon Berry \\ Technological University Dublin, damon.berry@tudublin.ie \\ Frank Duignan \\ Technological University Dublin, frank.duignan@tudublin.ie \\ Richard Hayes \\ Technological University Dublin, richard.hayes@tudublin.ie
}

Follow this and additional works at: https://arrow.tudublin.ie/teapotcon

Part of the Bioelectrical and Neuroengineering Commons, Biomedical Devices and Instrumentation Commons, Computer Engineering Commons, and the Other Biomedical Engineering and Bioengineering Commons

\section{Recommended Citation}

Berry, D. and Duignan, F. and Hayes, R.: An Investigation of the use of a High Resolution ADC as a "Digital Biopotential Amplifier. 4th European Conference of the International Federation for Medical and Biological Engineering. pp.142-147, 2009

This Conference Paper is brought to you for free and open access by the TPOT: People Oriented Technology at ARROW@TU Dublin. It has been accepted for inclusion in Conference Papers by an authorized administrator of ARROW@TU Dublin. For more information, please contact arrow.admin@tudublin.ie, aisling.coyne@tudublin.ie, gerard.connolly@tudublin.ie. Funder: self funding 


\title{
An Investigation of the use of a High Resolution ADC as a "Digital Biopotential Amplifier"
}

\author{
D. Berry ${ }^{1}$, F. Duignan ${ }^{1}$ and R. Hayes ${ }^{1}$ \\ ${ }_{1}$ Dublin Institute of Technology, tSchool of Electrical Engineering Systems, Dublin, Ireland
}

\begin{abstract}
Sigma delta analogue to digital converters have been used in many signal processing applications including some commercially available systems for sampling of bio-signals [1], [2]. Bio-potential amplifiers have traditionally utilised specialised highquality analogue components to amplify bio-signals.

The central aim of this work is to investigate the benefits of using high-resolution sigma delta conversion as a digital amplification stage in the signal path of a bio-potential amplifier. A number of system arrangements specific to bio-potential measurement are presented and discussed. These arrangements are designed to use the high-resolution capacity of the sigma delta converter in different ways, providing different benefits. To explore these benefits, the authors have implemented a digital bio-potential signal capture system consisting of a sigma delta converter interfaced to a PC running MATLAB via an inexpensive microcontroller and to test it with a number of test signals.

A signal generator which simulates the attributes of an Electrocardiogram was used together with analogue signal conditioning for two electrode and three electrode measurement to provide suitable signal sources for capture using the digital bioamplifier. The results were analysed to investigate the trade off between signal resolution and analogue amplification.
\end{abstract}

Keywords - 24-bit, sigma-delta, digital, biopotential, amplifier, ADC, analogue to digital converter.

\section{INTRODUCTION}

With the continuing reduction in cost, power consumption and size of digital systems comes the increasing opportunity for new implementations and new applications of existing solutions for biomedical instrumentation systems. The high resolution measurement capacity of the 24-bit Sigma-Delta analogue to digital converter [1], has been combined with suitably powerful digital signal processors to implement commercially available bio-amplification systems which benefit from the digitisation of the instrumentation system [2]. The aim of this work is to review the performance of this instrumentation combination with a view to (1) understanding and analysing the performance of a prototype system itself (2) investigating the cost and size of such a biosignal amplifier based on currently available components.

The aim of this work is to investigate the possible benefits and issues of sampling signals of low amplitude without analogue amplification by employing an inexpensive high resolution analogue to digital converter with suitable reference voltages. More specifically, the idea is to employ an embedded approach using inexpensive but powerful converters and microprocessors or microcontrollers to perform signal processing that would traditionally be performed by analogue amplifier and filter circuits. When this "digital amplifier" approach is incorporated into physiological measurement devices the signal passes through fewer components before it is converted into noise-immune digital form. The small number of components also means that it can be miniaturised and made portable.

The "digital amplifier" approach has been employed within various commercially available biosignal measurement systems. The authors have attempted to show that it is possible to develop a relatively inexpensive "digital amplifier" physiological recording system using off-theshelf components

\section{Measurement System OVERVIEW}

\section{A. Biopotential signals and their effects on the design of measurement systems}

Biopotential signals have amplitudes in the microvolt to millivolt range and spectral components of interest occur in the frequency range from $0.01 \mathrm{~Hz}$ to $10 \mathrm{kHz}$. Figure 1 adapted from Bronzino [3] shows approximate spectral ranges and amplitudes for three prominent types of biopotential signals.

To scale the signal from amplitudes of $\mathrm{mV}$ or even $\mu \mathrm{V}$ to the 3-10 V range at the input of a typical 12-bit ADC, the signal needs to be amplified. However, depending on the conditions of measurement, the d.c. electrode offset potential (referred to as "d.c. potential" in the graph and placed at $10^{-2} \mathrm{~Hz}$ for convenience) is often about $100 \mathrm{mV}$ and could be up to $1 \mathrm{~V}$ for non isolated systems [4] and unless it is dealt with this offset voltage can cause signal distortion or amplifier saturation when biosignals are amplified by typical gains of anything between 100 and 100,000 .

Table 1 shows some of the general properties of ECG and EEG measurement systems. Gains of over 1000 are generally required over anything from 3 to over 100 
channels. The resolution of the analogue to digital converter that is used in a bio-signal measurement system will depend on the application and can range upwards from 8 bits but 12 bits of resolution is typical. In the extreme case of a traditional 128 channel EEG measurement system with mostly discrete components, the chip count is significant.

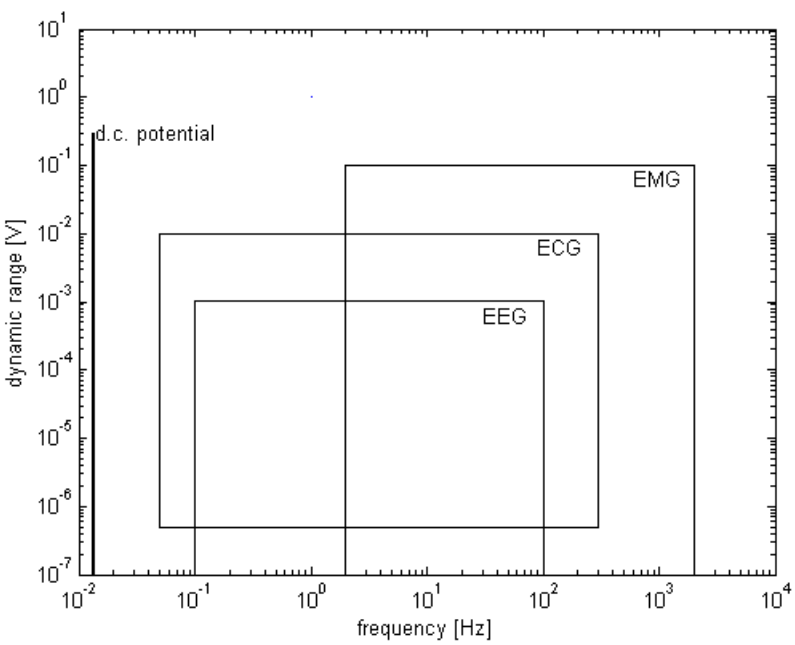

Figure 1: Dynamic ranges and spectral ranges for three types of bioelectric signals.

Table 1: Typical system characteristics of EEG and ECG signals.

\begin{tabular}{|l|l|l|l|l|l|l|l|}
\hline $\begin{array}{l}\text { Signal } \\
\text { Type }\end{array}$ & Amplitude & $\begin{array}{l}\text { Resolutio } \\
\mathrm{n} \\
{[\mathrm{per} \text { bit[] }}\end{array}$ & $\begin{array}{l}\text { Typical } \\
\text { Resolution } \\
{[\text { bits }]}\end{array}$ & $\begin{array}{l}\text { Bandwidth } \\
{[\mathrm{Hz}]}\end{array}$ & $\begin{array}{l}\text { Sample } \\
\text { Rate }\end{array}$ & $\begin{array}{l}\text { Number } \\
\text { of } \\
\text { Channels }\end{array}$ & $\begin{array}{l}\text { Gain for } \\
3 \mathrm{~V}\end{array}$ \\
\hline EEG & $5-300 \mu \mathrm{V}$ & $0.5 \mu \mathrm{V}$ & 9 & d.c. -150 & 300 & $3-100$ & 10,000 \\
\hline ECG & $0.5-4 \mathrm{mV}$ & $1-10 \mu \mathrm{V}$ & $8-12$ & $0.1-250$ & 500 & $3-12$ & 1000 \\
\hline
\end{tabular}

\section{B. Biopotential Amplifiers}

Electrobiological measurements traditionally employ a high quality analogue bio-potential amplifier which amplifies the differential mode signal between two or more electrodes. Due to the low amplitude of bio-potential signals, the differential mode signal is usually accompanied by comparatively high amplitude common mode noise caused by mains interference, and other bio-signals among other sources. Impedance mismatch between electrodes transforms a small portion of this common mode noise into differential mode noise which is difficult to separate from the desired bio-potential signal. Spinelli et. Al. [4] measured this transfer to be in the millivolt range for common mode voltages that were in volts.

The common mode noise can be reduced by using a combination of shielding or guarding [5] and a circuit arrangement known as 'driven right leg' [6], [7]. In shielding the common mode voltage is driven onto the protective sheath of the electrode cables and by employing a drivenright-leg. Driven right-leg is a particularly effective approach for common mode rejection which involves inverting and buffering the common mode voltage that appears at the inputs to the bio-potential amplifier and applying it to another electrode on the body of the subject. For electrocardiograms this electrode is affixed to the right leg. The input impedance of bio-potential amplifiers can vary from $10 \mathrm{M} \Omega$ for ECG amplifiers to $100 \mathrm{M} \Omega$ for EEG amplifiers. Two other key characteristics are the dynamic range which is the difference in scale from smallest to largest signal that the amplifier can respond to and the ability of the amplifier to recover from transients which may tend to 'swamp' the intended signal

\section{The Analogue to Digital Converter (ADC)}

The ADC chosen for this work was the ADS1256 from Texas Instruments.[8] This is a low cost, 24 bit (or less, depending on speed) Sigma Delta ADC with a Serial Peripheral Interface (SPI) for interconnection with a host microprocessor. The ADS1256 also incorporates a Programmable Gain Amplifier which facilitates "rangechanging" for different input signal ranges. There are 4 differential ( 8 single ended) inputs available.

The SPI interface of the ADS1256 allows a host processor to read and set values in internal configuration registers such as setting the actual sampling rate, controlling the multiplexer and reading converter status

\section{$D$. Interfacing the host processor to the $A D C$}

The processor chosen to interface to the ADC and to provide a communications bridge back to the PC was the Texas Instruments MSP430F149 processor [9]. This is a 16 bit 'RISC-like' processor that runs at $8 \mathrm{MHz}$. The Texas Instruments MSP processor family was designed with particularly low power consumption in mind, making it ideal for mobile, battery powered applications. The MSP430F149 also contains two serial interfaces facilitating its use as a bridge between a high speed serial interface to an ADC and an RS232 link to a PC. The MSP430F149 is a highly integrated processor so a very small number of components are required to produce an operational platform.

MSP430F149 connects to the ADS1256 using a $2 \mathrm{MHz}$ SPI link over which control and data information is sent. The MSP430F149 extracts ADC data from the ADS1256, reformats it into a format compatible with a data capture application running on the PC and then transmits it. The Microcontroller outputs the acquired data continuously via its UART1 serial port. A Matlab serial port read command 
is used to acquire the required number of samples which are saved to a file for later analysis. Results from a number of tests of the system are presented in the next section.

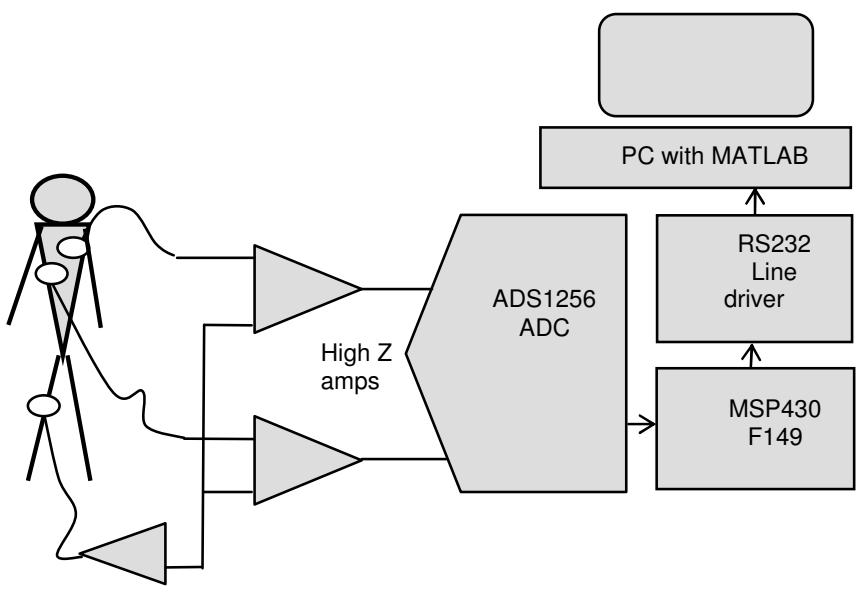

Figure 2 Block diagram of a digital biopotential amplifier incorporating a high resolution sigma delta ADC in three electrode mode.

\section{E. System Analysis and Test}

A simple test environment was used to prove the systems performance. A sampled ECG signal generated from an upsampled ECG record from the MIT-BIH Arrythmia database was sent out through a digital to analogue convertor (DAC) channel of an MSP430F439 microcontroller to produce signal to simulate an ECG signal. The DAC on the MSP430F439 has a reconstruction filter which smooths the edges on the analogue signal that is produced by the DAC. The signal from the DAC is then split and passed through different impedances to simulate different signal paths in the chest.

Figure 3 shows a block diagram of the test system. The (useful/noise-free) number of bits from the ADS1256 depends upon the sample rate and system noise. The ADS1256 samples internally at 30 thousand samples per second (ksps). The rate at which data is read from the device we shall call the actual sample rate. If the actual sample rate is $1 \mathrm{ksps}$ then each sample represents the average of 30 readings at the internal sample rate. Averaging changes the number of effective bits and so with an actual sample rate of less than $100 \mathrm{sps}$, the resolution is in the region of 24 bits. At $1 \mathrm{ksps}$, the number of effective bits drops to approximately 20 . The word 'approximately' is used because internal noise varies with configuration settings and so affects the number of useful bits in the output data.

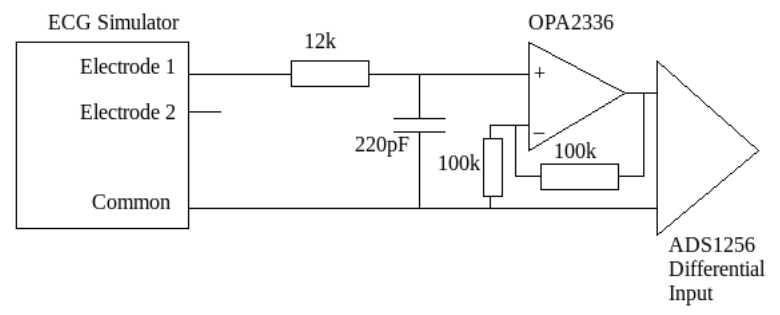

Figure 3a: The two electrode measurement system connecting a heartbeat simulator to a differential channel of the ADS1256

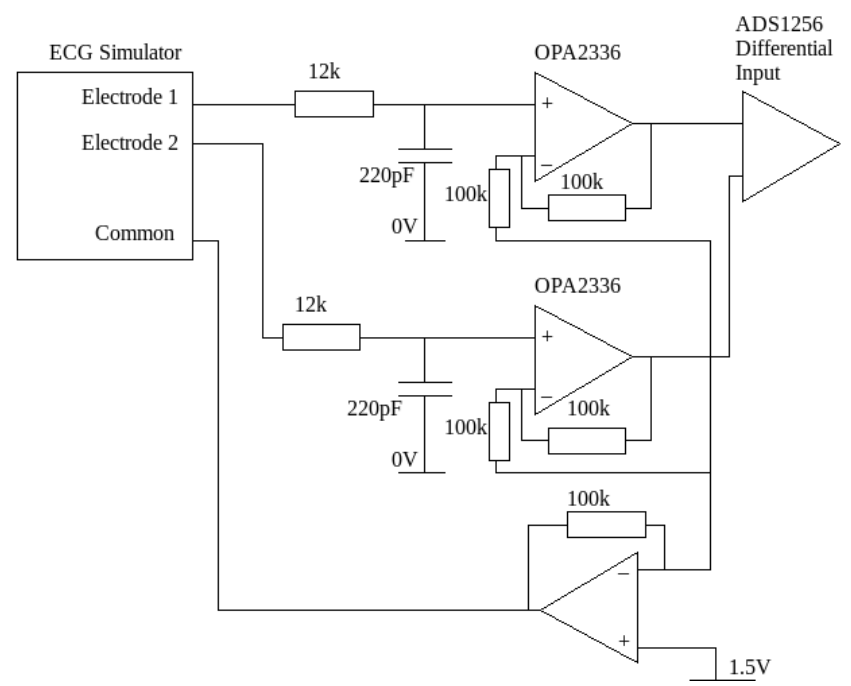

Figure 3b: The three electrode measurement system connecting a heartbeat simulator to a differential channel of the ADS1256.

Before looking at the test results it is worth considering the consequences of this variation in resolution, when using the ADS1256 to amplify and sample biosignals. Figure 4 shows the signal plane of the various biosignals this time superimposed on a graph showing the variation in resolution as a function of the Nyquist frequency of the converter. From the graph it can be seen that the sigma-delta converter can record most of the main features in the three types of biosignal. A limiting factor is the size of the electrode offset potential. In the absence of a high pass filter this would saturate once it is amplified towards the $3 \mathrm{~V} / 5 \mathrm{~V}$ limit of the ADS1256.

In order to sample and serially transmit the biopotential signals, a C-library was written to handle the interface between the MSP430F149 and ADS1256 and the PC. This is available for download on request from the authors.

The approach is similar to an approach that has been proposed in the Programmable Chip EEG project [10]. 


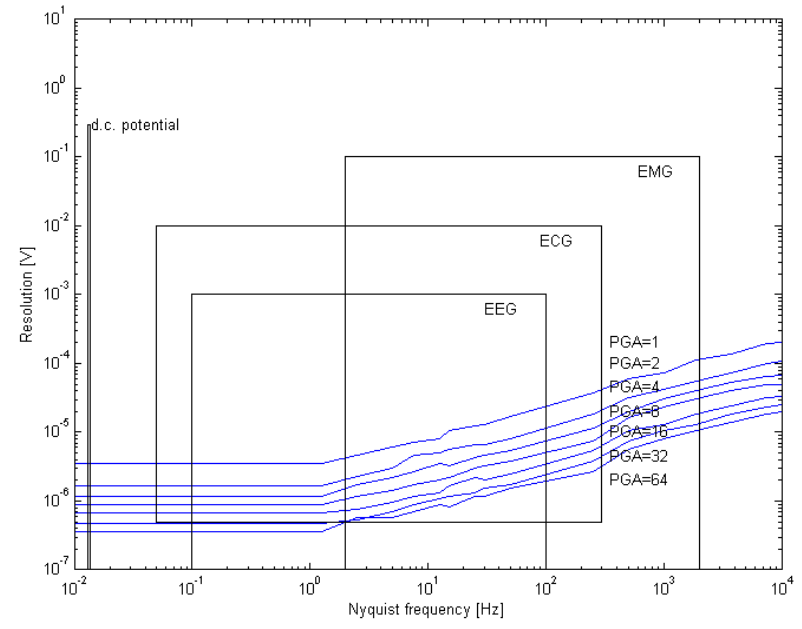

Figure 4: ADS1256 sigma delta converter resolution as a function of the Nyquist frequency corresponding to converter sample rates for different PGA gains and superimposed on the dynamic range and spectral ranges from Figure 1.

The results of the tests on the two configurations of the system are shown in Figure 5 and Figure 6. Figure 5 is composed of three discrete-time signals. The first part of the figure, figure 5a, shows an amplitude-normalized plot of the discretised data used to simulate the ECG signal in the heartbeat simulator. Figure $5 \mathrm{~b}$ is the amplitude-normalized ECG signal obtained from the two-lead measurement system, Figure $5 \mathrm{c}$ (the third trace) displays the normalised plot for the three-lead system. The difference in discrete time scaling is due to the different sampling rates used in the simulator and in the ADS1256-based measurement system which samples the analogue signal emitted by the digital to analogue convertor of the heartbeat simulator at a lower sampling rate.
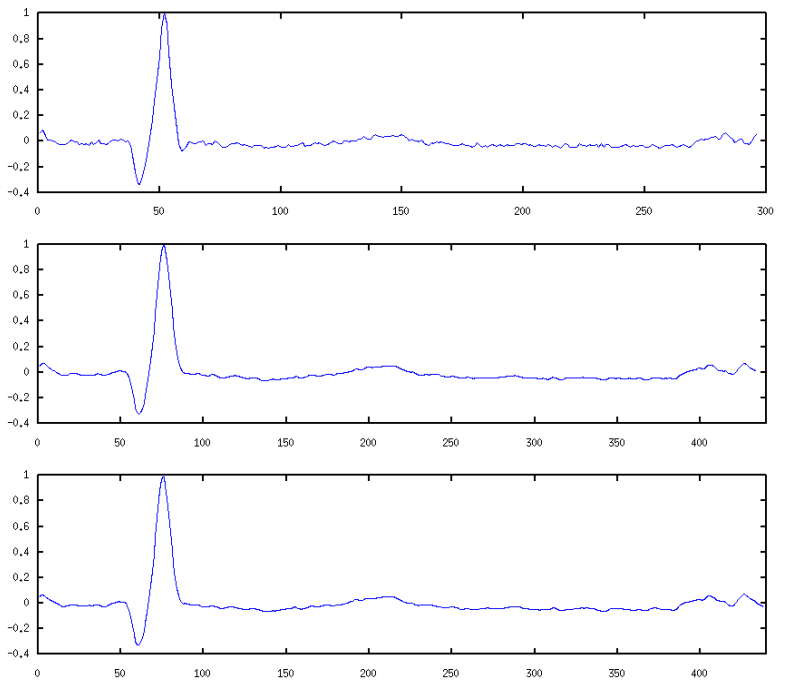

Figure 5. Discrete-time plots of the simulated ECG signal before it is sent to the ADC and the resulting sampled signal as measured for the two lead and three lead systems.

A comparison of the two output signals shows basic similarity between signals with little difference between the signals measured by the two lead and three lead systems although the amplitude of the non-normalized three-lead measurement is about $10 \%$ larger than the non normalized two-lead one.
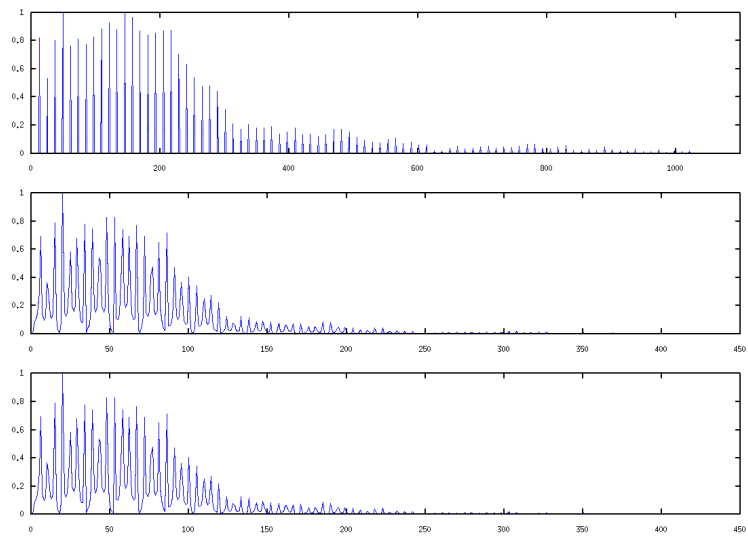

Figure 6: Fast fourier transform plots of the simulated ECG signal and the signals measured by the two lead and three lead configurations of the system

Figure 6 shows a fast fourier transform (FFT) of the three signals referred to above. Figure 6a shows the spectrum of the simulated signal, Figure $6 \mathrm{~b}$ shows the spectrum of the sampled signal from the two lead system and Figure 6c shows the spectrum from the three lead system. Again, the difference in frequency scaling is due to the difference in the sampling rates for simulator and measurement systems. The first peak is the fundamental frequency of the simulated heartbeat (at about 140bpm) and from comparison of the spectra it is apparent that up to frequency sample 800 on the simulated signal, the discrete spectra of the measured. This can be attributed to the low pass effect of the reconstruction filter at the output of the MSP430F439.

\section{Discussion}

This paper has proposed an experimental system for investigating the use of high resolution (up to 24-bits) analogue to digital convertors in physiological measurement applications. This prototype system has allowed the authors to embark on a range of experiments to investigate the benefits afforded by moving part of the required dynamic range of biopotential amplifiers into a digital environment. 


\section{A. Discussion of the relative merits of the digital amplifier approach over an analogue biopotential amplifier}

A sigma-delta analogue to digital convertor is a monolithic integrated circuit which although it may require supporting components when used in the front end of a digital biopotential amplifier. The large dynamic range $(120 \mathrm{~dB})$ of this type of converter at frequencies of up to $1 \mathrm{khz}$ suggest its use in the place of analogue circuits with similar dynamic range that are composed of a larger number of discrete components. The resulting miniaturisation allows the converter to be positioned close to the electrode and this in turn has the potential to improve signal to noise ratios.

Once the sampled voltage signal is converted to digital form, it is effectively immune to electrical noise and the sampled signal can be processed and transmitted digitally with no further loss of signal quality, thus providing a relatively stable environment for the signal. As discussed in section II, because the converter samples an unfiltered and unamplified signal in the presence of significant offset drift voltages, there is less risk of saturation of the sampled signal. It also reduces the need for analogue filtering of d.c. components. In addition, because the signal is converted into digital form at an early stage, digital isolation can be used to protect the user. Another benefit of using the sigma delta ADC is the ease of configuration.

While there is no doubt that the ADS1256 is rich in features, the authors have found that it is possible to define a relatively simple API which allows the user to configure the IC for multiple modes of operation at run time. The flexibility and stability of this digital configuration represents in the authors view an improvement over the potential difficulties associated with configuration of exclusively analogue bioamplifier circuits.

In effect, the use of a digital amplifier results in a tradeoff; the switch from analogue to digital signal processing requires less complex circuitry at the expense of increased computational load. The combination of microprocessor and converter acting on the unfiltered signal introduces the possibility of incorporating software self calibration and range changing functionality. The input impedance of the analogue input pins on the ADS1256 with the input buffer enabled is $40 \mathrm{M} \Omega$ at $500 \mathrm{~Hz}$ [8] this input impedance is lower than impedances found on biopotential amplifiers which require input impedances of at least $100 \mathrm{M} \Omega$. This problem can be solved by the introduction of a very high impedance amplifier configured as a unity gain buffer for each electrode.

Like many analogue to digital converters, the ADS 1256 employs a multiplexer to switch analogue input channels and so does not sample its input pins simultaneously. Even in the case of differential inputs this results in a phase delay between the sampled versions of the two sides of the signal. One way of overcoming this difficulty would be to tie multiple converters to the same signal lines so that the conversions are synchronised. The authors will investigate this aspect in future work.

It has been mentioned in section II that some physiological amplifiers employ a driven right leg feature where the common mode voltage is inverted and superimposed on the reference voltage. The availability of inexpensive 20-bit digital to analogue converters raises the possibility of developing a "digital driven right leg" or low amplitude perturbation signals which could be used to characterise the electrical properties of the body.

\section{B. Cost and Size of the proposed system}

24-bit sigma delta analogue to digital converters such as the multi-channel ADS 1256 are inexpensive, they retail for less than $€ 10$. Although the two electrode digital biopotential amplifier presented here could not by any means be considered a complete system, with a component count of five components and comparatively low cost in the authors' view it represents a compact and inexpensive way to 'amplify' a bioelectrical signal.

\section{CONCLUSIONS}

This paper has presented an investigation of the use of a low cost, low power consumption combination of a high resolution analogue to digital converter and microcontroller to implement an inexpensive, robust and easily maintained biopotential measurement channel. A working prototype has been demonstrated and initial results have been presented. This system has been developed with a view to its implementation in specialized lower cost portable ambulatory biopotential measure systems.

\section{AcKNOWLEDGMENT}

The authors would like to thank DIT engineering graduate students Aisling Cronin and Rupal Patel and students of the ME in Advanced Engineering at DIT for their initial investigations and experiments on the characteristics of biosignals and on the ADS1256 Sigma Delta Convertor which helped pave the way for this work.

\section{REFERENCES}

1. Jarman D.(1995) Intersil Corporation Application Note AN9504 A brief introduction to sigma delta conversion www.intersil.com

2. www.biosemi.com 
3. Bronzino (2006) The Biomedical Engineering Handbook: Medical Devices and Systems CRC Press

4. Spinelli E. Mayosky, M.A. Pallas Areny R., (2006) A practical approach to electrode-skin impedance unbalance measurement pp1451-1453 IEEE transactions on biomedical engineering 53:14511453

5. MettingVanRijn, A.C., Peper A., Grimbergen C.A., (1990) High quality recording of bioelectric events: Interference reduction, theory and practice Med Biol Eng Comput, 28:5:389-97

6. Winter B.B. and Webster J.G. (1983) Driven-right-leg circuit design IEEE Trans. Biomedical Eng. Vol. BME-30 1:62 -
7. Winter B.B. Webster J.G. (1983) Reduction of interference due to common mode voltage in biopotential amplifiers IEEE Trans. Biomedical Eng.Vol. BME-30:1:58-61

8. Texas Instruments (2006) ADS1256 very low noise 24-Bit analogueto-digital converter Technical Note SBAS2881 www.ti.com

9. Texas Instruments (2004) MSP430x13x, MSP430x14x, MSP430x14x1 Mixed Signal Microcontroller Data Sheet \#SLAS272F June 2004 www.ti.com

10. http//www.opencircuits.com/Programmable_Chip_EEG 